\title{
The Effects of Aquatic Watsu Therapy on Gross Motor Performance and Quality of Life for Children with Cerebral Palsy
}

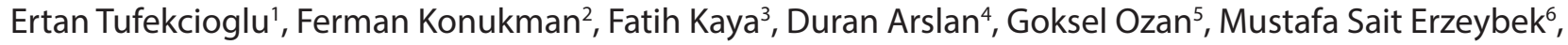 \\ Esam A. Al-Sawi
} Affiliations: ${ }^{1}$ King Fahd University of Petroleum \& Minerals, College of Applied Studies, Physical Education Department, Dammam, Saudi Arabia, ${ }^{2}$ Qatar
University, College of Arts and Sciences, Sport Science Program, Doha, Qatar, ${ }^{3}$ Erzincan Binali Yildırım University, Faculty of Education, Department of
Physical Education and Sports, Erzincan, Turkey, ${ }^{4}$ Youth and Sports Ministry, Istanbul, Turkey, ${ }^{5}$ Marmara University, Faculty of Sport Sciences, Department
of Physical Education and Sports, Istanbul, Turkey, ${ }^{6}$ Dumlupinar University, Faculty of Sport Sciences, Department of Physical Education and Sports, Kutahya, Turkey, ${ }^{7}$ King Fahd University of Petroleum \& Minerals, College of Sciences, Department of Mathematics and Statistics, Dammam, Saudi Arabia

Correspondence: F. Konukman, Qatar University, College of Arts and Sciences, Sport Science Program, PO. Box. 2713, Doha, Qatar. E-mail: fkonukman@ qu.edu.qa

\begin{abstract}
The purpose of this study was to compare the effects of Immersion and Watsu ${ }^{\circledR}$ therapy protocols on children with cerebral palsy. Gross motor function (GMF), spasticity (MAS), and Quality of Life (QoL) parameters of twenty-three children (age: 7.5 $\pm 2.8, \mathrm{BMI}: 17 \pm 3.7$ ) were measured. Subjects received Watsu ${ }^{\circledR}$ therapy and Immersion protocols in Watsu-Immersion (W-I, $\mathrm{n}=12)$ and Immersion-Watsu $(\mathrm{I}-\mathrm{W} \mathrm{n}=11)$ groups in different periods based on a crossover design. The subjects received sessions for 30 minutes twice a week during 10-week experimental periods. The results were investigated with independent sample t-test and Wilcoxon rank-sum tests, which showed that there was no evidence of carryover effects in GMFM 88 and QoL. In contrast, Watsu ${ }^{\circledR}$ improved GMFM 88 (p<0.05), Quality of Life $(p<0.05)$, and MAS-Upper spasticity $(p<0.05)$, scores significantly compared to immersion. The current results demonstrated the specific benefits of Watsu ${ }^{\circledast}$ therapy on children with $\mathrm{CP}$, confirming the previous anecdotal reports. We recommend Watsu ${ }^{\circledR}$ as a safe and well-accepted complementary intervention for the management of $C P$.
\end{abstract}

Keywords: Watsu ${ }^{\oplus}$, cerebral palsy, immersion, motor function, quality of life

@MJSSMontenegro

THE EFFECTS OF AQUATIC WATSU THERAPY

http://mjssm.me/?sekcija=article\&artid=219

Cite this article: Tufekcioglu, E., Konukman, F., Kaya, F., Arslan, D., Ozan, G., Erzeybek, M.S., \& Al-Sawi, E.A. (2021). The Effects of Aquatic Watsu Therapy on Gross Motor Performance and Quality of Life for Children with Cerebral Palsy. Montenegrin Journal of Sports Science and Medicine, 10 (2), 25-30. doi: 10.26773/mjssm.210904

\section{Introduction}

Cerebral palsy $(\mathrm{CP})$ is the most common cause of childhood-onset, lifelong motor disability with the prevalence rate of about 2.1 per 1,000 live births in developed countries. The Center for Disease Control and Prevention stated that 1 in 321 children had been classified with $\mathrm{CP}$, and it is found to be more common in boys than in girls (CDC, 2018).

As a result of non-progressive brain lesions, developmental neuromuscular and musculoskeletal disorders in $\mathrm{CP}$ are the result of neural impairments deteriorating the homeostat- 
ic state, the capacity for participation and the quality of life (QoL) (Hadders-Algra. 2014).

The constraints in CP are reduced joint mobility, contractures and bone deformities, abnormal motor control, and dependence on external support in daily life (Blair \& Cans, 2018). Although physical and occupational therapy improves functional abilities at various stages of development, motor impairment, abnormal movement patterns, and postural deformities can deteriorate due to the lack of interventions over time (Blair \& Cans, 2018). The coordinated and multidisciplinary management of $\mathrm{CP}$ focuses on the QoL to improve neuromuscular and musculoskeletal complications and prognosis (Hadders-Algra, 2014).

$\mathrm{CP}$ can cause a dramatic decrease in the physical activity level. Neuro-motor, socio-emotional, cognitive, and musculoskeletal conditions are the area of focus in the increasing number of aquatic studies (Carayannopoulos et al., 2020; Schitter et al., 2020). Many aquatic interventions are being investigated due to its feasibility and minimum adverse effects (Roostaei et al., 2017). In addition to the many other benefits observed, aquatic interventions have improved functional motor skills, mobility, strength, tension in the muscles, and inflammation (Choi \& Cho, 2019; Lai, 2014).

Relatively new in the history of aquatic therapy, Watsu, is a hands-on, client-centred approach that seeks to improve relaxation, muscle tone in the sense of security, and promote trust in which the client can surrender him/herself to the water and the therapist. Watsu is recognized in rehabilitation for the variety of conditions (Brody \& Geigle, 2009) including fibromyalgia, neurologic conditions, physical function, chronic disabilities, and lower back pain (Schitter et al., 2020). The gentle Watsu mobilization can enhance body awareness (Schitter \& Fleckenstein, 2018). Although, its practicality, time and cost-efficiency benefits, Watsu is one of the least investigated forms of aquatic intervention; therefore, generalizing of the results for the variety of disabilities impossible.

The challenge in CP is the low level of QoL associated with gross motor dysfunction. QoL determines the physical and psychological wellbeing, self-perception, and pain levels. It can be improved with therapeutic and motor control interventions (Muñoz-Blanco et al., 2020).

Spasticity is expressed as a velocity-dependent increase in muscle tone, hyperreflexia, and the presence of primitive reflexes that can result in abnormal posture, delayed motor development, and atypical gait patterns (Li \& Francisco, 2019). Neurological disorders can change the muscle tone dramatically. Holistic aquatic intervention studies on spasticity can provide insight for the aetiology and contribute to forming better multimodal treatment settings.
In brief, cost-effective modalities are essential in $\mathrm{CP}$ due to lifelong expensive health care. Therefore, we compared and evaluated the effectiveness of Watsu and Immersion on QoL, motor function and spasticity in $\mathrm{CP}$.

\section{Methods}

The study was conducted in two private rehabilitation facilities that provide health care and social services to disabled children in Istanbul, Turkey. A crossover design was conducted in comparison to the effect of Watsu and Immersion. All subjects were assigned to either Watsu-Immersion (W-I) or Immersion-Watsu (I-W) groups, according to aged-stratified randomization.

The standardized manual was used to perform gross motor function measurement (GMFM) 88, MAS and CP QoL, Version July 2, 2013, Primary Caregiver Questionnaire 4-12 years) tests at baseline and upon completion of the periods. We used CP QoL Child, an assessment to obtain insight into several aspects of children's lives (Waters et al., 2013) as a parent-reported QoL assessment correlating with the impairment (Arnaud et al., 2008). The GMFM 88 is an observational clinical tool designed to assess the level of impairments, motor skills and evaluate the improvement more accurately than GMFM 66 (Ko \& Kim, 2013). The validity (Josenby et al., 2009) and reliability (Russell et al., 2000) of GMFM-88 were shown. The most common spasticity measure, modified Aschworth scale (MAS) was used. It is a six-point rating scale developed as a simple clinical classification to assess muscle tone (Ward et al., 2016).

The Deanship of Scientific Research at King Fahd University of Petroleum and Mineral, Dhahran, Saudi Arabia, approved the research protocol.

\section{Subjects}

We assigned children with no seizures in the month before the study into two groups based on aged-stratified randomization with no significant differences in their descriptive data. There were 11 female and 12 male subjects aged $4-12(\mathrm{~N}=23$, age $7.52 \pm 2.78$, weight $17.29 \pm 4.03$, height $94.83 \pm 11.26$, BMI 19.04 \pm 1.62 ). Informed consent was obtained from all the subjects included in the study. Their parents provided informed consent forms and confirmed the participation of their children for the publishing of the outcomes. The subjects with no systemic condition finished their health check-up before the study. The gross motor function classification system (GMFCS) levels and type of CP (topographic distribution) were shown (Table 1). The subjects were able to understand basic verbal commands and report sensitivity to pain.

Table 1. The GMFCS levels and topographic distribution of the subjects

\begin{tabular}{|c|c|c|c|c|c|c|c|c|}
\hline \multirow{2}{*}{ Groups } & \multirow{2}{*}{$\mathrm{N}$} & \multicolumn{2}{|c|}{ Gender } & \multirow{2}{*}{ Age } & \multicolumn{2}{|c|}{ Limb distribution } & \multicolumn{2}{|c|}{ GMFCS } \\
\hline & & $\mathrm{F}$ & M & & Diplegia & Hemiplegia & 1 & II \\
\hline W-I & 12 & 7 & 5 & $7.92(3.11)$ & 1 & 11 & 6 & 6 \\
\hline $\mathrm{I}-\mathrm{W}$ & 11 & 4 & 7 & $7.09(2.42)$ & 1 & 10 & 5 & 6 \\
\hline
\end{tabular}

\section{Exclusion Criteria}

The exclusion criteria were the previous surgical procedure for saliva control, drugs taken influencing saliva secretion and cardiac modulation at least 15 days before the test for the study. Subjects with pathologies associated with infectious or viral states, inability to maintain an orthostatic position, medical history of cardiac diseases and any diseases known to affect the autonomic cardiac function (neurological, endocrine disorders) were not involved. Absenteeism and fatigue were the reasons for exclusion after the beginning of the experimental phase (Figure 1). 


\section{Recruited \\ Randomized \\ Dropped-Out \\ Completed}

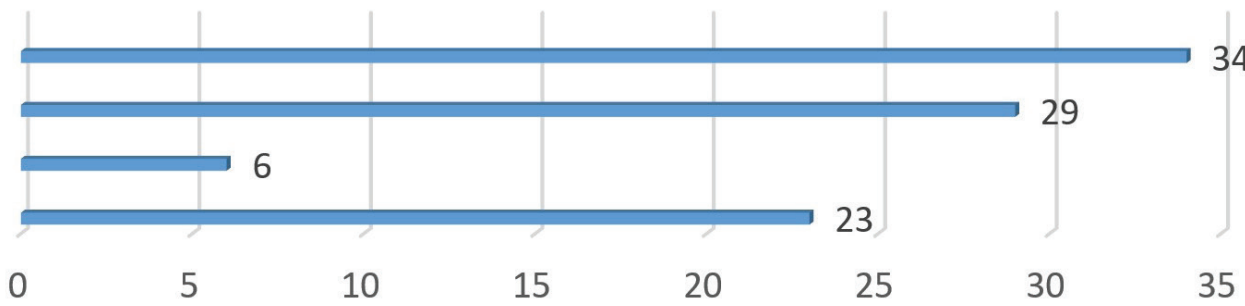

Figure 1. The total numbers of participants during the course of the study

\section{Experimental Design}

Experts used the standardized manual to perform GMFM 88, QoL and MAS, tests at baseline and upon completion of the periods. W-I group $(\mathrm{n}=12)$ received Watsu, whereas I-W group $(n=11)$ received immersion in the first period (Figure 2). No aquatic intervention therapy, medications, or surgical procedures were applied in the six weeks of washout interval between the periods.

In the second period, the groups went from receiving Watsu to Immersion and from Immersion to Watsu therapy. Two certified practitioners experienced in the health care of children were in charge of the application of the adapted Watsu.

\section{Intervention Protocol}

In the Watsu therapy protocol, the bodies of the children were manipulated, stretched, and mobilized in the form of gentle twists, rocking and cradling movements together with massage and pressure point work. The 30-minute Watsu or immersion sessions were applied twice a week for ten weeks.
Both groups received Watsu and immersion in different periods. The sessions consisted of spine-rotating techniques, such as slow offering, leg offerings, spiral offering, free spine, accordion, rotating accordion, twist-over, corner-spread and chest-opening, seaweed. The Watsu therapists started with less impaired and less stiff side of the children with gentle support from under knees to keep the hips and knees flexed. They focused first on spinal movements progressing to the limbs and avoided stretching multiple joints into extension at the same time.

A constant intention was given to performing traction and rotational movements considering the limitations of children. In immersion, the back of the subject's head and neck was supported by specific floating equipment allowing the rest of the body to remain in the water. No technique was applied while children were closely observed. The temperature of the pool water and air were $33^{\circ} \mathrm{C}$ and $28^{\circ} \mathrm{C}$, respectively. The post-intervention data collection was identical to the baseline procedure.

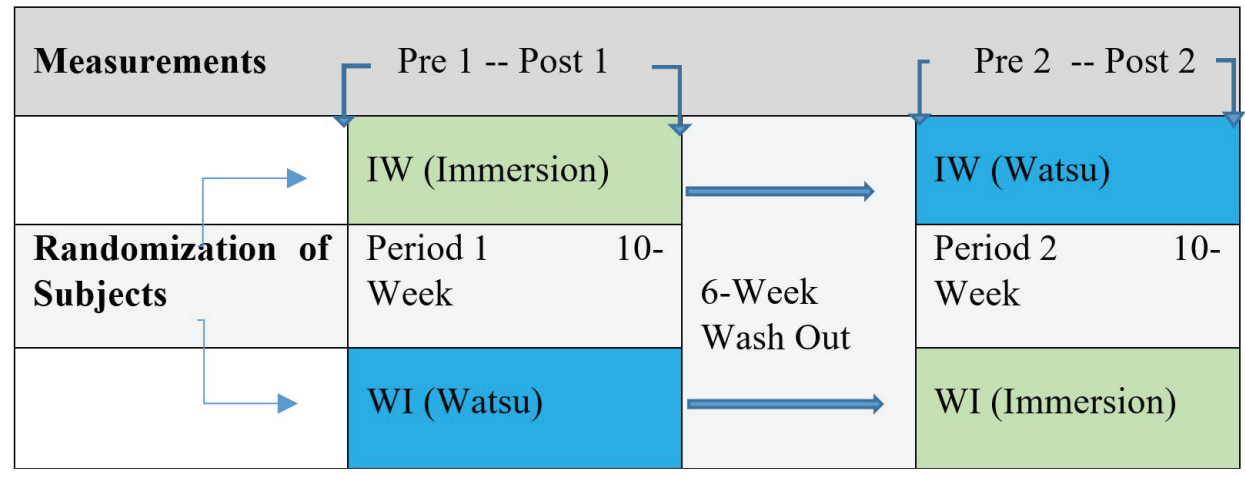

Figure 2. The cross over design for this study

\section{Statistical Analysis}

The sum of the measured values in both periods for each subject was calculated and compared with the independent sample t-test to clarify the effectiveness of the washout period in eliminating any carryover effect. In the case of significant results, no comparison was performed between the therapy differences. The therapeutic effects were analysed using the first period values. Paired t-tests and Independent t-tests were used to compare the difference within and between the groups. In the case of no carryover effect, the effects were evaluated based on the difference between the two therapies and the independent sample t-test was used. Wilcoxon rank-sum test and test procedures similar to those mentioned above were used in order to analyse ordinal data. The level of significance for all tests was set at $5 \%(\mathrm{p}<0.05)$.

\section{Results}

The subject's descriptive data (whole sample) are presented in Table 2. None of their physical characteristics differences was significant $(\mathrm{p}>0.05)$.

Table 2. Physical characteristics of the groups - Mean (SD)

\begin{tabular}{ccccc}
\hline Groups & Age (year) & Weight $(\mathrm{kg})$ & Height $(\mathrm{cm})$ & $\mathrm{BMI}\left(\mathrm{kg} / \mathrm{m}^{2}\right)$ \\
\hline W-I $(\mathrm{n}=12)$ & $7.92(3.12)$ & $17.92(4.56)$ & $96.58(12.52)$ & $18.99(1.77)$ \\
$\mathrm{I}-\mathrm{W}(\mathrm{n}=11)$ & $7.09(2.43)$ & $16.61(3.44)$ & $92.91(9.95)$ & $19.09(1.53)$ \\
Total $(\mathrm{N}=23)$ & $7.52(2.78)$ & $17.29(4.03)$ & $94.83(11.26)$ & $19.04(1.62)$ \\
\hline
\end{tabular}


Watsu improved GMFM 88 significantly compared to immersion $(t=6.698, p=0.000)$. No evidence of relevant car- ryover effects in GMFM $88(\mathrm{t}=-1.386, \mathrm{p}=0.180)$ was found, (Table 3).

Table 3. GMFM 88 measurement results of the subjects - Mean (SD)

\begin{tabular}{cccccc}
\hline Groups & Gross Motor & \multicolumn{2}{c}{ Period 1 } & \multicolumn{2}{c}{ Period 2 } \\
\hline W-I & GMFM 88 & $0.037(0.011)$ & $0.039(0.010)$ & $0.037(0.011)$ & $0.038(0.010)$ \\
I-W & GMFM 88 & $0.031(0.010)$ & $0.032(0.009)$ & $0.031(0.010)$ & $0.033(0.010)$ \\
\hline
\end{tabular}

Watsu improved QoL significantly compared to Immersion $(\mathrm{t}=2.212, \mathrm{p}=0.038)$. No relevant carryover effects were seen in

QoL ( $\mathrm{t}=1.555, \mathrm{p}=0.135)$, (Table 4).

Upper Spasticity after Watsu was significantly better com-

Table 4. Quality of life questionnaire results of the subjects - Mean (SD)

\begin{tabular}{cccccc}
\hline Groups & Quality of Life & \multicolumn{2}{c}{ Period 1 } & \multicolumn{2}{c}{ Period 2 } \\
\hline W-I & QoL & $64.78(2.81)$ & $65.42(2.57)$ & $64.75(2.83)$ & $64.92(2.84)$ \\
I-W & QoL & $64.36(3.61)$ & $64.45(3.45)$ & $64.36(3.44)$ & $64.55(3.45)$ \\
\hline
\end{tabular}

pared to Immersion $(Z=-2.222, p=0.026)$. No carryover effect was

lower spasticity more than immersion did $(\mathrm{Z}=-1.025, \mathrm{p}=0.305)$. seen in Upper Spasticity $(\mathrm{Z}=-1.125, \mathrm{p}=0.261)$. Watsu improved the There were carryover effects $(\mathrm{Z}=-2.009, \mathrm{p}=0.045)$, (Table 5$)$.

Table 5. Modified Ashworth Scale scores of the subjects - Mean (SD)

\begin{tabular}{|c|c|c|c|c|c|}
\hline \multirow{2}{*}{$\frac{\text { Groups }}{\text { W-I }}$} & \multirow{2}{*}{$\frac{\text { MAS }}{\text { Upper Spasticity }}$} & \multicolumn{2}{|c|}{ Period 1} & \multicolumn{2}{|c|}{ Period 2} \\
\hline & & $1.47(1.35)$ & $1.42(1.33)$ & $1.47(1.35)$ & $1.47(1.35)$ \\
\hline $\mathrm{I}-\mathrm{W}$ & & $2.09(.86)$ & $2.06(.89)$ & $2.09(.86)$ & $1.97(.91)$ \\
\hline W-I & Lower Spasticity & $2.15(.84)$ & $2.06(.89)$ & $2.12(.88)$ & $2.12(.88)$ \\
\hline $\mathrm{I}-\mathrm{W}$ & & $2.57(.91)$ & $2.57(.91)$ & $2.54(.90)$ & $2.30(.91)$ \\
\hline
\end{tabular}

\section{Discussion}

The initial evidence for the effect of Watsu on GMFM 88, QoL and MAS indices were provided by this current study (Table 3, 4, 5), in line with previous studies suggesting the benefits of aquatic therapies on various areas of functioning (Roostaei et al., 2017; Lai et al., 2014).

The varying intervention approaches ranging from passive to active and physical to psychological are needed to provide sufficient amounts of afferent input (Scott et al., 2020). Thus, the altered visual, tactile, proprioceptive and vestibular inputs in Watsu therapy may have played a critical role in the reorganization of deteriorated sensory, neuromotor skills, which form the basis for motor functioning and muscular tonus improvements (Tables 3,5 ). Also, the activated proprioceptive track, neuromuscular and afferent $\mathrm{C}$-tactile fibre stimulation in warm water can reinstate the earlier stages of ontogeny, reducing motor function constraints (Barassi et al., 2018). Vestibular input and activation of afferent C-tactile fibres can also alleviate hypertonia that results in higher GMFM 88.

Moreover, sensory-motor inputs due to the enriched environment and bodywork could have improved not only GMF but also emotional regulation in CP (Meireles et al., 2017; Lai et al., 2014). Thus, integrated Watsu movements used to improve the individualization of the sessions and the thermal conductive properties of water can meet the needs in specific areas, including muscular tonus reduction (Table 5). As tonus reduces, the therapist could perform techniques more efficiently that can provide a higher quality of tactile and proprioceptive input with increased range of motion. This increase in ROM and efficiency can optimize body structures and re-educate the internal reference systems (Novak, 2014).

The kinesthetic sensations in Watsu from stretches, flexion and extension of the limbs and the torso, traction and rotation of the spine are critical for the control of movement and can improve conscious influences on motor function, thus, GMFM 88 (Meireles, 2017). Watsu may have enhanced the accordance between proprioception and kinesthesia. Moreover, the use of a single leg or arm can improve awareness of laterality, which in turn, can improve motor output.

The close presence of the trusted therapist can likely cause feelings of security and relaxation, which can result in the ease of expressions of the feelings. The reduced protective muscle guarding and tonus might be the reflection of this potential. Therefore, the increased vestibular, tactile, and proprioceptive input to the cortico-limbic system in the form of mobilization of joints and manipulation and elongation of muscles might have contributed to the reduction in upper spasticity (Table 5). We believe that altered cardiac afferent input has played a role in the reduced tonus (Amichai \& Katz-Leurer, 2014). Thus, the majority of techniques provided while two torsos are in close contact could have enhanced the sensory-motor afferent input improving spasticity. Moreover, a specific Watsu session can be implemented with a focus on either upper or lower limbs, considering the need of each child. Therefore, better spasticity levels and independence, adaptive behaviours, social participation and QoL might be achieved.

Many variables determine QoL (Noor et al., 2015), which requires a multidisciplinary approach (Novak, 2014). However, improved QoL implies the multidimensional wellbeing efficacy of Watsu (Table 4). The activation of afferent sensory visceral inputs to the cortico-limbic system and regulation of somatic efferent neuronal motor pathways are linked to the regulation of emotional state (Kinner et al., 2014), thereby, QoL. In the current study, the increased QoL might be rooted in the altered sensorimotor experience in the form of gentle movements, stretching, and trigger point work in 
Watsu (Dull, 2008).

Nevertheless, QoL is linked to the psychosocial development of children and psychological stress that require a proper parental attitude (Al-Dababneh \& Al Zboon, 2018; Arnaud et al., 2008). Parental dysfunction and stress can lead to the severity of the disorder and additional problems including, parents' reduced psychological adjustment, the mothers' QoL, and the way in which parents report QoL of their children (Noor et al., 2015). Families need to direct their attention to their personal needs in order to maintain day-to-day management of their responsibilities. The family members can also join and receive the therapy with their children to release parental stress while contributing to a variety of socio-emotional gains for their children that may improve QoL.

Moreover, the desired results require parental support as much as therapists do. Family-centred intervention services in health care can contribute to greater independence at a lowcost and time-efficient way. Intensive training is recommended for providing a variety of relaxing and emotional quality of touch, considering Watsu applications in the home environment. However, the families can apply the basic form of the therapy after having entry-level training. Therefore, the more engaged the families are, the better the understanding of the children's capabilities the families have. As a result, the family-centred care environment in improving motor function and QoL are supported (Kokorelias et al., 2019).

The results illuminated the potential extrapolations for future studies to achieve functional and structural adaptations using Watsu. Therefore, we suggest Watsu, as a versatile therapy to be incorporated in the aquatic rehabilitation programs for $\mathrm{CP}$, considering no common side effects and family engagement possibilities. Nevertheless, establishing well-rounded physiotherapy practises from our study is limited.

\section{Conclusion}

The current study provides initial evidence in favour of Watsu over immersion and suggests it as a complementary method to improve motor functions, QoL and spasticity in CP. This initial evidence can form a basis for the future large-scale studies comparing the efficacy of Watsu and its subdivisions with other aquatic interventions to achieve a higher QoL and enhanced motor function. In this regard, despite the promising findings of the current study, further research is needed to investigate the potential of Watsu on more diverse samples in the form of isolated therapy for individuals and community-based group therapy. The respiratory deficiency, emotional regulation, and participation or community integration can also be investigated considering the potential influence on physiologic and psychological parameters. Thus, the confirmed versatility of Watsu can help health professionals to utilize Watsu in different pediatric aquatic therapy protocols more efficiently. Lastly, longer follow-up periods and randomized controlled design studies are needed in future studies to confirm the effect of Watsu across age categories and GMFCS levels.

There are several limitations to this study. First, the possible influences of the diet were not evaluated. Second, the Water Orientation Test Alyn2 for aquatic skills assessment was not used. Third, parental stress and QoL were not measured simultaneously. Fourth, follow-up measurements were not performed.

Finally, the holistic approach of Watsu can enrich inter- disciplinary CP treatment in water. Participation of individualized Watsu sessions may be a more attainable and attractive goal than taking conventional aquatic therapy.

\section{Acknowledgement}

We gratefully acknowledge the support provided by Deanship of Scientific Research at King Fahd University of Petroleum \& Minerals through Project IN 151004 for this research. All experimental procedures and methods were explained in detail to each parent, who then signed the Informed Consent Forms approved by the Deanship of Scientific Research at KFUPM.

\section{References}

Al-Dababneh, K. A., \& Al-Zboon, E. K. (2018). Parents' attitudes towards their children with cerebral palsy. Early Child Development and Care, 188(6), 731-47. doi: 10.1080/03004430.2016.1230737

Amichai, T., \& Katz-Leurer M. (2014). Heart rate variability in children with cerebral palsy: Review of the literature and meta-analysis. Neurorehabilitation, 35(1), 113-122.

Arnaud, C., White-Koning, M., Michelsen, S. I., Parkes, J., Parkinson, K., Thyen, U., Beckung, E., Dickinson, H. O., Fauconnier, J., Marcelli, M., McManus, V., \& Colver, A. (2008). Parent-reported quality of life of children with cerebral palsy in Europe. Pediatrics, 121(1), 54-64. doi: 10.1542/peds.2007-0854.

Banky, M., Ryan, H. K., Clark, R., Olver, J., \&Williams, G. (2017). Do clinical tests of spasticity accurately reflect muscle function during walking: A systematic review, Brain Injury, 31(4), 440-455. doi: 10.1080/02699052.2016.1271455

Barassi, G., Bellomo, R. G., Porreca, A., Saggini, R., Irace, G., Giannuzzo, G., \& Trivisano, L. (2018). Rehabilitation of neuromotor disabilities in aquatic microgravity environment. Advances in Experimental Medicine and Biology, 1113, 61-73. doi: 10.1007/5584_2018_164

Blair, E., \& Cans, C. (2018). The definition of cerebral palsy. In C. P. Panteliadis (Ed.), Cerebral Palsy: A Multidisciplinary Approach, Third Edition (pp. 13-17). Switzerland: Springer International Publishing AG. doi: 10.1007/978-3-31967858-0_2

Brody, L. T., \& Geigle, P. R. (2009). Aquatic exercise for rehabilitation and training. Champaign. IL: Human Kinetics.

Carayannopoulos, A. G., Han, A., \& Burdenko, I. N. (2020). The benefits of combining water and land-based therapy. Journal of Exercise Rehabilitation, 16(1), 20-26. doi: $10.12965 /$ jer.1938742.371

Centers for Disease Control and Prevention. (2018). Data and statistics of Cerebral Palsy. Retrieved from https://www. cdc.gov/ncbddd/cp/data.html

Choi, K., \& Cho, S. (2019). The effect of underwater intervention in children with cerebral palsy on the range of motion, coordination and balance of motion: metaanalysis. Korean Journal of Integrated Medicine, 7(3), 7183. doi: 10.15268/KSIM.2019.7.3.071

Dull H. (2008). Watsu: basic and explorer paths. Watsu Publishing.

Hadders-Algra, M. (2014). Early diagnosis and early intervention in cerebral palsy. Frontiers in Neurology, 5, 185. doi: 10.3389/fneur.2014.00185

Josenby, A.L., Jarnlo, G. B., Gummesson, C., \& Nordmark, E. 
(2009). Longitudinal construct validity of the GMFM-88 total score and goal total score and the GMFM-66 score in a 5-year follow-up study, Physical Therapy, 89(4) 342-350. doi: 10.2522/ptj.20080037

Kinner, V. L., Het, S., \& Wolf, O. T. (2014). Emotion regulation: exploring the impact of stress and sex. Frontiers in Behavioral Neuroscience, 8, 397. doi:10.3389/ fnbeh.2014.00397

Ko, J., \& Kim, M. (2013). Reliability and responsiveness of the Gross Motor Function Measure- 88 in children with cerebral palsy. Physical Therapy, 93(3). 393-400. doi: 10.2522/ptj.20110374.

Kokorelias, K. M., Gignac, M. A. M., Naglie, G., \& Cameron, J. I. (2019).Towards a universal model of family centered care: A scoping review. BMC Health Service Research, 19, 564. doi: 10.1186/s12913-019-4394-5

Lai, C., Liu, W. Y., Yang, T. F., Chen, C., Wu, C. Y., \& Chan, R. C. (2014). Pediatric aquatic therapy on motor function and enjoyment in children diagnosed with cerebral palsy of various motor severities. Journal of Child Neurology, 30(2), 200-8. doi: 10.1177/0883073814535491

Li, S., \& Francisco, G. E. (2019) Spasticity. In: Handbook of Experimental Pharmacology. Springer, Berlin, Heidelberg.

Meireles, A. L., Marques, M. R., Segabinazi, E., Spindler, C., Piazza, F. V., Salvalaggio, G. S., Augustin, O. A., Achaval, M., \& Marcuzzo, S. (2017). Association of environmental enrichment and locomotor stimulation in a rodent model of cerebral palsy: Insights of biological mechanisms. Brain Research Bulletin, 128, 58-67. doi:10.1016/j.brainresbull

Muñoz-Blanco, E., Merino-Andrés, J., Aguilar-Soto, B., García, Y. C., Puente-Villalba, M., Pérez-Corrales, J., \& GüeitaRodríguez, J. (2020). Influence of aquatic therapy in children and youth with cerebral palsy: A qualitative case study in a special education school. International Journal of Environmental Research and Public Health, 17, 3690.

Noor, R., Gul, S., Saleem, T., \& Shahzad, N. (2105). Relationship of Stress, Anxiety and Psychological Adjustment among Parents of Intellectually Disabled and Cerebral Palsy Children. International Journal of Rehabilitation Science, $4(2), 32$.
Novak, I. (2014). Evidence-based diagnosis, health Care, and rehabilitation for children with cerebral palsy. Journal of Child Neurology. 29(8), 1141-56. doi: 10.1177/0883073814535503

Roostaei, M., Baharlouei, H., Azadi, H., \& Fragala-Pinkham, M. A. (2017). Effects of aquatic intervention on Gross Motor Skills in children with cerebral palsy: A systematic review. Physical Occupational Therapy Pediatric, 37(5), 496-515. doi:10.1080/01942638.2016.1247938

Russell, D. J., Avery, L. M., Rosenbaum, P. L., P. S. Raina, S. D. Walter., \& R. J. Palisano. (2000). Improved scaling of the Gross Motor Function measure for children with cerebral palsy: Evidence of reliability and validity. Physical Therapy, 80, 873-875.

Schitter, A. M., \& Fleckenstein, J. (2018). Passive hydrotherapy WATSU $^{\circledR}$ for rehabilitation of an accident survivor: A prospective case report. Complementary Medicine Research, 25(4), 263-268. doi: 10.1159/000487768

Schitter, A. M., Fleckenstein, J., Frei, P., Taeymans, J., Kurpiers, N., \& Radlinger, L. (2020). Applications, indications, and effects of passive hydrotherapy WATSU (WaterShiatsu) - A systematic review and meta-analysis. PLoS ONE, 15(3): e0229705

Scott, J., Wozencroft, A., Nocera, V., Webb, K., Anderson, J., Blankenburg, A., Watson, D., \& Lowe, S. (2020). "Aquatic Therapy Interventions and Disability: A recreational therapy perspective." International Journal of Aquatic Research and Education, 12(3), 5. doi:10.25035/ ijare.12.03.05

Ward, R., Reynolds, J. E., Bear, N., Elliott, C., \& Valentine, J. (2016). What is the evidence for managing tone in young children with, or at risk of developing cerebral palsy: a systematic review. Disability, and Rehabilitation, 58(5), 492-501. doi: 10.3109/09638288.2016.1153162

Waters, E., Davis, E., Boyd, R., Reddihough, D., Mackinnon, A., Graham, H. K., Lo, S. K., Wolfe, R., Stevenson, R., Bjornson, K., Blair, E., \& Ravens-Sieberer, U. (2013). Cerebral Palsy Quality of Life Questionnaire for Children (CP QOL-Child) Manual. Melbourne: University of Melbourne. 